\title{
Utilização de carvão ativado de dendê in natura e funcionalizado em meio ácido na adsorção de paracetamol
}

\author{
Application of activated carbon dendê in \\ nature and functionalized on amid acid \\ in paracetamol adsorption
}

\author{
Regiane Cristina Ferreira ${ }^{1}$, Hugo Henrique Carline de Lima ${ }^{2}$, Osório Moreira Couto Junior ${ }^{1}$, \\ Pedro Augusto Arroyo ${ }^{1}$, Karina Querne de Carvalho ${ }^{3}$, \\ Maria Angélica Simões Dornellas de Barros ${ }^{1}$
}

\footnotetext{
${ }^{1}$ Departamento de Engenharia Química - DEQ/ UEM CEP: 87.020-900, Maringá, PR

${ }^{2}$ Departamento de Química - DQI/ UEM CEP: 87.020-900, Maringá, PR

${ }^{3}$ Departamento Acadêmico de Construção Civil - DACOC/UTFPR CEP: 81.280-340, Curitiba, PR e-mail: regiane.cristina.1989@gmail.com, hugolima22@hotmail.com,osorio_eq@yahoo.com.br, arroyo.deq@gmail.com, kaquerne@utfpr.edu.br, angelicabarros.deq@gmail.com
}

\begin{abstract}
RESUMO
Uma possível solução para remoção dos fármacos nos corpos hídricos é por meio da adsorção com carvão ativado. O objetivo do trabalho foi estudar a utilização de carvão de dendê in natura e funcionalizado com ácido nítrico $\left(\mathrm{HNO}_{3}\right)$ na adsorção do paracetamol. As amostras foram caracterizadas quanto às propriedades texturais e químicas. Ensaios cinéticos e de equilíbrio foram realizados com ambas as amostras nos valores de $\mathrm{pH}$ : 2,0, $\mathrm{pH}_{\mathrm{PCZ}}$ e 11,0 a $25{ }^{\circ} \mathrm{C}$. Um bloqueio dos microporos parece ter ocorrido no carvão ativado funcionalizado por grupos funcionais de óxido introduzidos pelo tratamento químico, acarretando na diminuição da eficiência de remoção do paracetamol. No método de Boehm observou-se aumento de grupos ácidos e diminuição nos valores do $\mathrm{pH}_{\mathrm{PCZ}}$. $\mathrm{O}$ modelo cinético de pseudo-segunda ordem e o modelo de equilíbrio de Langmuir e Sips se ajustaram melhor aos dados experimentais. Maior quantidade adsorvida do paracetamol foi verificada nos ensaios com $\mathrm{pH}$ 2,0 e $\mathrm{pH}_{\mathrm{PCZ}}$ para os carvões quando comparado aos ensaios com $\mathrm{pH}$ 11,0, devido à repulsão eletrostática.
\end{abstract}

Palavras-chave: fármaco, carvão nacional, tratamento químico.

\begin{abstract}
A possible solution for the removal of pharmaceuticals in water bodies is by means of adsorption with activated carbon. The objective of this work was to study the utilization of dendê carbon in nature and functionalized with nitric acid $\left(\mathrm{HNO}_{3}\right)$ in the paracetamol adsorption. The samples were characterized as the textural and chemical properties. Kinetic and equilibrium assays were performed with both samples at $\mathrm{pH}$ values: 2.0 , $\mathrm{pH}_{\mathrm{PZC}}, 11.0$ at $25^{\circ} \mathrm{C}$. Micropore blocking seemed to take place in the functionalized activated carbon by oxide groups introduced by the chemical treatment, thus causing decrease in the removal efficiency of paracetamol. In the Boehm method was observed an increase in the acid groups and a decrease in the $\mathrm{pH}_{\mathrm{PZC}}$ values. The kinetic model of pseudo-second order and the equilibrium Langmuir and Sips model were best fitted to the experimental data. Greatest amount of paracetamol adsorbed was noted in the assays with $\mathrm{pH} 2.0$ and $\mathrm{pH}_{\mathrm{PZC}}$ to the carbons when compared to the assays with $\mathrm{pH} 11.0$, due to electrostatic repulsion.
\end{abstract}

Keywords: pharmaceutical, Brazilian carbon, chemical treatment.

\section{INTRODUÇÃO}

O descarte de esgotos sanitários in natura nos cursos de água tem sido foco de contaminação dos recursos hídricos por medicamentos e produtos de higiene e limpeza, visto que em muitas localidades há déficit de infraestrutura em saneamento. Outra fonte de contaminação é o lançamento de efluentes de estações de tra- 
tamento de esgotos sanitários, uma vez que os fármacos são substâncias recalcitrantes aos processos convencionais de tratamento [1].

Estudos mais recentes tem sido reportados sobre a detecção da presença de fármacos como ácido acetilsalicílico e salicílico, amlodipina, cafeína, carbamazepina, cetoprofeno, ciprofloxacina, diclofenaco, esparfloxacina, gemifloxacina, ibuprofeno, moxifloxacina, naproxeno, ofloxacina, paracetamol e rosuvastatina em águas superficiais e subterrâneas, indicando a limitação dos sistemas de tratamento de esgotos na remoção destes compostos recalcitrantes que podem se tornar um risco à saúde humana e ao ambiente aquático [2, 3, 4]. Embora as concentrações dos fármacos ainda sejam vestigiais no ambiente, acredita-se que estes possam atingir concentrações potencialmente tóxicas devido ao seu crescente consumo.

Infelizmente não há tratamento específico para remoção de fármacos, e é neste contexto que se insere a adsorção, com destaque para o uso do carvão ativado de origem vegetal como adsorvente por suas características químicas e texturais.

Até o presente momento, alguns poucos trabalhos de pesquisa foram reportados sobre remoção do paracetamol por adsorção [5] em carvões ativados in natura e funcionalizado. A funcionalização do carvão ativado pode levar a modificações significativas no mecanismo e na seletividade da adsorção, criando um leque de possibilidades de estudos dos mecanismos e das condições ótimas de operação que irão proporcionar conhecimento mais aprofundado sobre as variáveis intervenientes no processo de adsorção [6].

Neste contexto, o objetivo deste trabalho foi comparar a eficiência da remoção do paracetamol pela adsorção em amostras de carvão de cascas de coco de dendê in natura e funcionalizadas com ácido nítrico.

\section{MATERIAIS E MÉTODOS}

\subsection{Adsorvato}

Paracetamol ou acetaminofeno (N-(4-hidroxifenil)etanamida), nomenclatura popular do N-acetil-paminofenol, foi sintetizado pela Sigma-Aldrich (pureza de 98\%). A concentração inicial para os ensaios cinéticos e isotermas de adsorção foi de $3,30 \times 10^{-4} \mathrm{~mol} \mathrm{~L}^{-1}$ do paracetamol com $10 \%$ metanol em água ultrapura obtida de sistemas de purificação de água Milli-Q.

\subsection{Adsorventes}

O adsorvente utilizado foi carvão de casca de coco de dendê cedido pela Bahiacarbon (Bahia, Brasil). Primeiramente, as amostras foram lavadas com água deionizada a temperatura de $50{ }^{\circ} \mathrm{C}$, secas em incubadora durante $24 \mathrm{~h}$ a $60^{\circ} \mathrm{C}$ até completa remoção da umidade, moídas e peneiradas a 80 mesh ASTM.

Para funcionalização com ácido nítrico foram utilizados $10 \mathrm{~g}$ de carvão ativado mantidas em agitação de $288 \mathrm{rpm}$ a $60{ }^{\circ} \mathrm{C}$ com $100 \mathrm{~mL}$ de solução de $\mathrm{HNO}_{3}$ a $1 \mathrm{~mol} \mathrm{~L}{ }^{-1}$ por $24 \mathrm{~h}$ [7]. Após este tratamento, o excesso do $\mathrm{HNO}_{3}$ foi eliminado por meio de lavagens das amostras com água deionizada. Em seguida, as amostras do carvão funcionalizado foram mantidas em estufa a $45^{\circ} \mathrm{C}$ durante $48 \mathrm{~h}$.

As amostras do carvão in natura (DD) e funcionalizado com ácido nítrico (DAC) foram submetidas à caracterização e a ensaios de adsorção em batelada conforme descrito nas seções 2.3 e 2.4. As soluções de paracetamol usadas nos ensaios cinéticos e de equilíbrio com as amostras dos carvões foram preparadas com $\mathrm{pH} 2,0, \mathrm{pH}_{\text {PZC }}$ e 11,0 usando soluções de $\mathrm{NaOH}$ e $\mathrm{HCl} 0,1 \mathrm{~mol} \mathrm{~L}^{-1}$.

\subsection{Caracterização}

As amostras dos carvões foram caracterizadas por adsorção e dessorção de $\mathrm{N}_{2}$, utilizando o equipamento Micromeritics, EUA, modelo ASAP 2020 a $-196{ }^{\circ} \mathrm{C}$ e $0{ }^{\circ} \mathrm{C}$ com $\mathrm{N}_{2}$ líquido. Antes da caracterização, as amostras foram desgaseificadas à vácuo $\left(10^{-2} \mathrm{~Pa}\right)$ durante $2 \mathrm{~h}$ a $300^{\circ} \mathrm{C}$. Foram obtidas as respectivas áreas superficiais pelo método de Brunauer-Emmett-Teller $\left(\mathrm{A}_{\mathrm{BET}}\right)$ na faixa de $0,05<\mathrm{P} / \mathrm{P}_{0}<0,30$, volume de microporos $\left(\mathrm{V}_{\alpha \mathrm{T} \text { - }}\right.$ tal ) pelo método t-plot e o volume total dos poros $\left(\mathrm{V}_{\text {Total }}\right)$. $\mathrm{O}$ volume de mesoporos $\left(\mathrm{V}_{\text {Meso }}\right)$ foi calculado como diferença entre $\mathrm{V}_{\text {Total }}$ e $\mathrm{V}_{\alpha \text { Total }}$ o volume de ultramicroporos $\left(\mathrm{V}_{\alpha \text { Ultra }}\right.$ ) pelo método AlphaS ( $\alpha$ s) e o volume de supermicroporos $\left(\mathrm{V}_{\alpha \text { Super }}\right)$ pela diferença entre o $\mathrm{V}_{\alpha \text { Total }}$ e $\mathrm{V}_{\alpha \text { Ultra }}$.

$\mathrm{O}$ procedimento de determinação do ponto de carga zero $\left(\mathrm{pH}_{\mathrm{PCZ}}\right)$ foi baseado no trabalho de Park e Regabulto [8]. A determinação dos grupos funcionais foi realizada de acordo com o método de Boehm [9]. A análise de espectroscopia no infravermelho foi feita em um espectrofotômetro da BRUKER, modelo Vertex $70 \mathrm{v}$ com leitura de comprimento de onda da transmitância na faixa de 400-4000 $\mathrm{cm}^{-1}$ com resolução de $2 \mathrm{~cm}^{-1}$. As amostras foram secas em estufa a $100{ }^{\circ} \mathrm{C}$ durante $12 \mathrm{~h}$, e posteriormente misturadas com $\mathrm{KBr}$ na proporção de $0,5 \%$ de adsorvente por $99,5 \%$ de $\mathrm{KBr}$. 


\subsection{Ensaios Cinéticos}

As cinéticas de adsorção foram estudadas por meio do contato de $10 \mathrm{mg}$ dos carvões com $20 \mathrm{~mL}$ da solução de paracetamol sob agitação de $150 \mathrm{rpm}$ a temperatura controlada de $25^{\circ} \mathrm{C}$ em banho termostatizado Dubnoff da Nova Ética, com coleta de amostras no intervalo de tempo de 5 a 480 minutos no pH de 2,0, $\mathrm{pH}_{\mathrm{PCZ}}$ e 11,0. Após atingido o tempo previamente especificado, as amostras foram filtradas em papel filtro qualitativo (Unifil, faixa azul, diâmetro de $125 \mathrm{~mm}$ ), e efetuadas as leituras da concentração do fármaco em espectrofotômetro uv-vis Hach no comprimento de onda de $245 \mathrm{~nm}$ [10]. Os ensaios foram realizados em triplicata. A quantidade adsorvida foi determinada por balanço de massa como mostrado na Equação 1.

$$
q_{t}=\frac{\left(C_{0}-C_{a}\right) V}{M}
$$

Em que: $q_{t}$ é a quantidade adsorvida no tempo $t\left(\mathrm{mg} \mathrm{g}^{-1}\right) ; C_{0}$ e $C_{a}$ correspondem a concentração do paracetamol na fase líquida inicial e no tempo t, respectivamente $\left(\mathrm{mg} \mathrm{L}^{-1}\right) ; V$ é o volume da solução (L); e $M$ é a massa (g) de sorvente.

Os dados experimentais das curvas cinéticas ( $\mathrm{q}_{\mathrm{t}}$ versus $t$ ) foram ajustados ao modelo de pseudoprimeira ordem [11], conforme apresentado na Equação 2.

$$
\frac{d q_{t}}{d t}=k_{1}\left(q_{e}-q_{t}\right)
$$

Em que: $q_{t}$ é a quantidade de moléculas adsorvidas $\left(\mathrm{mg} \mathrm{g}^{-1}\right)$ em tempos diferentes; $q_{e}$ é a quantidade de moléculas adsorvidas em equilíbrio $\left(\mathrm{mg} \mathrm{g}^{-1}\right)$; e $k_{1}$ é a constante do modelo cinético de pseudo-primeira ordem $\left(\mathrm{min}^{-1}\right)$. Para obter a equação na forma linearizada, integrou-se a Equação 2 de $\mathrm{t}=0$ a t $=\mathrm{t}$ e de $q_{t}=0$ a $q_{t}=q_{t}$ e obteve-se a Equação 3 . Neste caso, $\mathrm{k}_{1}$ e $\mathrm{q}_{\mathrm{e}}$ foram obtidos pelo ajuste não linear no gráfico $q_{e}$ versus t.

$$
q_{t}=q_{e} \times\left(1-e^{-k_{1} t}\right)
$$

Os dados experimentais das curvas cinéticas também foram ajustados ao modelo de pseudo-segunda ordem [12], conforme apresentado na Equação 4.

$$
\frac{d q_{t}}{d t}=k_{2}\left(q_{e}-q_{t}\right)^{2}
$$

Em que: $k_{2}$ é a constante do modelo cinético de pseudo-segunda ordem $\left(\mathrm{g} \mathrm{mg}^{-1} \mathrm{~min}^{-1}\right)$.

Integrando a Equação 4, com a condição inicial $q_{t}=0$ em $\mathrm{t}=0$, tem-se a Equação 5.

$$
q_{t}=\frac{q_{e}^{2} \times k_{2} \times t}{1+k_{2} \times q_{e} \times t}
$$

Os valores de $\mathrm{k}_{2}$ e $\mathrm{q}_{\mathrm{e}}$ foram obtidos pelo ajuste não linear no gráfico $q_{t}$ versus $\mathrm{t}$.

\subsection{Isotermas}

As isotermas de adsorção foram obtidas por meio do contato de diferentes massas dos carvões, variando de 8 a $50 \mathrm{mg}$, com solução do paracetamol em concentração inicial fixa $\left(3,30 \times 10^{-4} \mathrm{~mol} \mathrm{~L}^{-1}\right)$ para os valores de $\mathrm{pH}$ $2,0, \mathrm{pH}_{\mathrm{PCZ}}$ e 11,0 . Os ensaios foram realizados em banho termostático a $25^{\circ} \mathrm{C}$ durante 240 minutos, sob agitação a $150 \mathrm{rpm}$. As suspensões foram filtradas em papel filtro qualitativo (Unifil, faixa azul, diâmero de 125 $\mathrm{mm}$ ) e analisadas no espectrofotômetro uv-vis Hach DR 5000 a $245 \mathrm{~nm}$.

Com os resultados experimentais foram construídos os gráficos de $q_{e}$ versus $C_{e}$ e ajustados aos modelos de isotermas de Langmuir [13], Freundlich [14] e Sips [15], conforme apresentado no Quadro 1. 
Tabela 1: Equações dos modelos de Langmuir, Freundlich e Sips.

\begin{tabular}{|c|c|c|}
\hline Modelo & Equação & Variáveis \\
\hline Langmuir & $q_{e}=\frac{q_{\max } \times C_{e}}{1+k_{L} \times C_{e}}$ & $\begin{array}{l}q_{e} \text { é a quantidade de molécula adsorvida em equilíbrio com so- } \\
\left.\text { lução por unidade de massa (g de adsorvato g de adsorvente }{ }^{-1}\right) \text {; } \\
q_{\max } \text { é a quantidade máxima de molécula adsorvida em solução } \\
\text { por unidade de massa para formar monocamada completa de } \\
\left.\text { adsorção (g de adsorvato g de adsorvente }{ }^{-1}\right) ; k_{L} \text { é a constante da } \\
\text { isoterma de Langmuir, relacionada com a energia de adsorção ( } \mathrm{L} \\
\left.\mathrm{mg}^{-1}\right) ; C_{e} \text { é a concentração do adsorvente na fase líquida não } \\
\text { adsorvida em equilíbrio }\left(\mathrm{g} \mathrm{L}^{-1}\right) \text {. Os parâmetros } k_{L} \text { e } q_{\max } \text { são de- } \\
\text { terminados a partir dos dados experimentais. }\end{array}$ \\
\hline Freundlich & $q_{e}=k_{F} \times C_{e^{\frac{1}{n F}}}$ & $\begin{array}{l}k_{F} \text { e } 1 / n_{F} \text { são constantes que dependem de diversos fatores expe- } \\
\text { rimentais e se relacionam com a distribuição dos sítios ativos e a } \\
\text { capacidade de adsorção do adsorvente [1]. }\end{array}$ \\
\hline Sips & $q_{e}=\frac{q_{\max } \times\left(k_{S \times} C_{e}\right)^{n}}{1+\left(k_{S \times} C_{e}\right)^{n}}$ & $\begin{array}{l}k_{S} \text { é a constante da isoterma de Sips, com a energia de adsorção } \\
\left(\mathrm{L} \mathrm{mg} \mathrm{m}^{-1}\right) ; n \text { representa o grau de heterogeneidade do sistema, } \\
\text { podendo variar de } 0 \text { a } 1 \text {. Se } n=1 \text { o sistema é homogêneo, igua- } \\
\text { lando-se ao modelo de Langmuir e } n<1 \text { representa aumento da } \\
\text { heterogeneidade. Os parâmetros } q_{\text {máx }}, k_{S}, n \text { são determinados a } \\
\text { partir da análise de regressão não-linear. }\end{array}$ \\
\hline
\end{tabular}

\section{RESULTADOS E DISCUSSÕES}

\subsection{Caracterização}

Na Figura 1 são apresentadas as isotermas de adsorção e dessorção de $\mathrm{N}_{2}$ para as amostras dos carvões ativados estudados. As isotermas de adsorção e dessorção de $\mathrm{N}_{2}$ são classificadas de acordo com classificação da IUPAC (2015) [16] como sendo do tipo I (b), caracterizada com materiais que têm distribuições de tamanho de poro em uma ampla faixa, incluindo microporos mais largos e possivelmente mesoporos estreitos, com histerese do tipo H4, característica de materiais micro-mesoporosos.

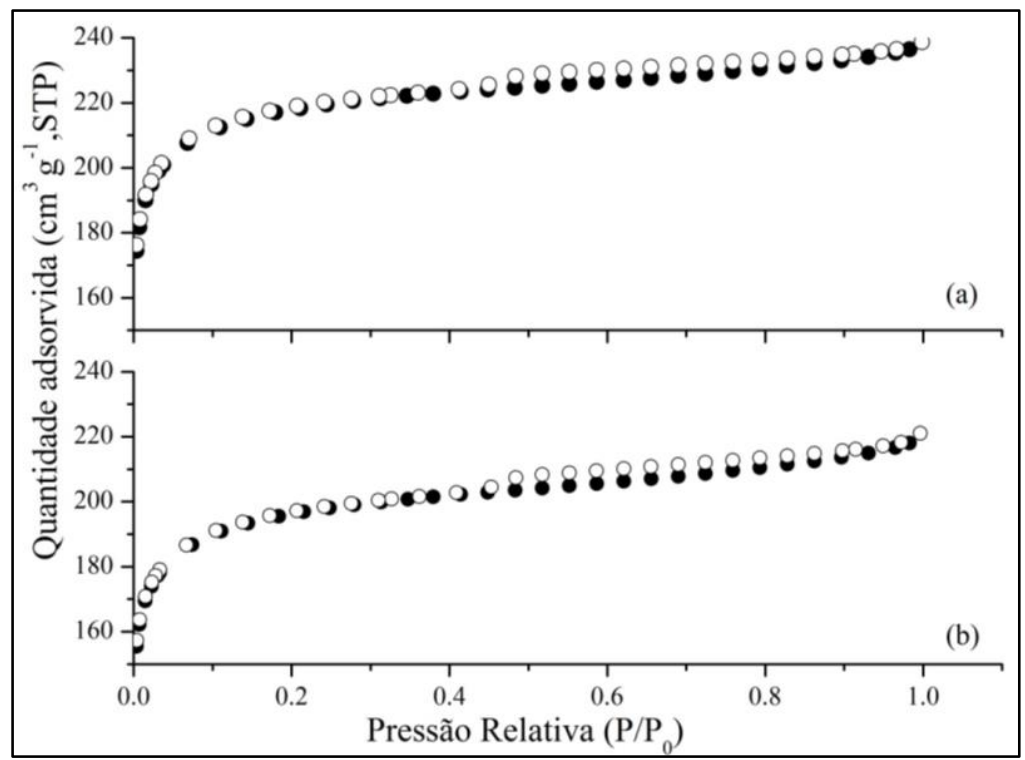

Figura 1: Isotermas de $(\mathbf{)})$ adsorção e $(\bigcirc)$ dessorção de $\mathrm{N}_{2}$ dos carvões ativados (a) DD e (b) DAC.

Os resultados da caracterização textural dos carvões ativados das propriedade área superficial, volume de microporos, volume total dos poros, volume de mesoporos, volume de ultramicroporos e volume de supermicroporos são apresentadas na Tabela 1 . 
Tabela 1: Propriedades texturais dos carvões ativados estudados.

\begin{tabular}{ccccccc}
\hline Amostra & $\begin{array}{c}\mathbf{A}_{\text {BET }} \\
\left(\mathrm{m}^{2} \mathrm{~g}^{-1}\right)\end{array}$ & $\begin{array}{c}\mathbf{V}_{\text {Total }}{ }^{* *} \\
\left(\mathrm{~cm}^{3} \mathrm{~g}^{-1}\right)\end{array}$ & $\begin{array}{c}\mathbf{V}_{\text {Meso }}{ }^{* *} \\
\left(\mathrm{~cm}^{3} \mathrm{~g}^{-1}\right)\end{array}$ & \multicolumn{3}{c}{ Método $\boldsymbol{\alpha}_{\mathbf{s}}\left(\mathbf{c m}^{3} \mathbf{g}^{-1}\right)$} \\
\hline DD & 671,90 & 0,37 & 0,08 & 0,29 & 0,20 & 0,09 \\
\hline DAC & 622,57 & 0,35 & 0,09 & 0,26 & 0,19 & 0,07 \\
\hline
\end{tabular}

Verifica-se que a funcionalização promoveu diminuição da área específica e do volume de microporos e parece não ter afetado o volume de mesoporos nas amostras in natura. Nas amostras do carvão tratado com $\mathrm{HNO}_{3}$ ocorreu diminuição de 7,35\% na área BET. Resultado este inferior aos notados por Lopes [17] que observou redução na $\mathrm{A}_{\mathrm{BET}}$ de $12,4 \%$ (de 803,43 para 703,66 $\mathrm{m}^{2} \mathrm{~g}^{-1}$ ) com carvão tratado por $\mathrm{HNO}_{3}(6 \mathrm{M}$ ).

De fato, o tratamento ácido com $\mathrm{HNO}_{3}$ tem efeito de penetrar nos poros dos carvões, sendo possível verificar considerável diminuição na sua área específica [18]. Os resultados também estão de acordo com os apresentados por Shim [19] e Fernandes [20].

Os resultados do método de Boehm e do $\mathrm{pH}_{\mathrm{PCZ}}$ são apresentados na Tabela 2.

Tabela 2: Grupos básicos, carboxílicos, lactônicos e fenólicos dos adsorventes (em mEq g ${ }^{-1}$ ) e $\mathrm{pH}_{\mathrm{pcz}}$.

\begin{tabular}{ccccccc}
\hline Amostra & $\begin{array}{c}\text { Grupos } \\
\text { Carboxílicos* }\end{array}$ & $\begin{array}{c}\text { Grupos } \\
\text { Lactônicos* }\end{array}$ & $\begin{array}{c}\text { Grupos } \\
\text { Fenólicos* }\end{array}$ & $\begin{array}{c}\text { Grupos Totais } \\
\text { ácidos* }\end{array}$ & $\begin{array}{c}\text { Grupos } \\
\text { básicos* }^{*}\end{array}$ & pH $_{\mathbf{p c z}}$ \\
\hline DD & 0,1752 & 0 & 0 & 0,1752 & 0,7000 & 6,5 \\
\hline DAC & 1,1014 & 0 & 0,2220 & 1,3234 & 0,5732 & 3,6 \\
\hline
\end{tabular}

Segundo Julien [21] tratamentos com ácido nítrico reagem com grupos básicos como cromenos e pironas, formando grupos ácidos por abertura dos heterociclos e consequentemente reduzindo o $\mathrm{pH}$ dos carvões, o que foi verificado no $\mathrm{pH}_{\mathrm{PCZ}}$ do carvão funcionalizado neste trabalho.

Os espectros no infravermelho obtidos estão apresentados na Figura 2.

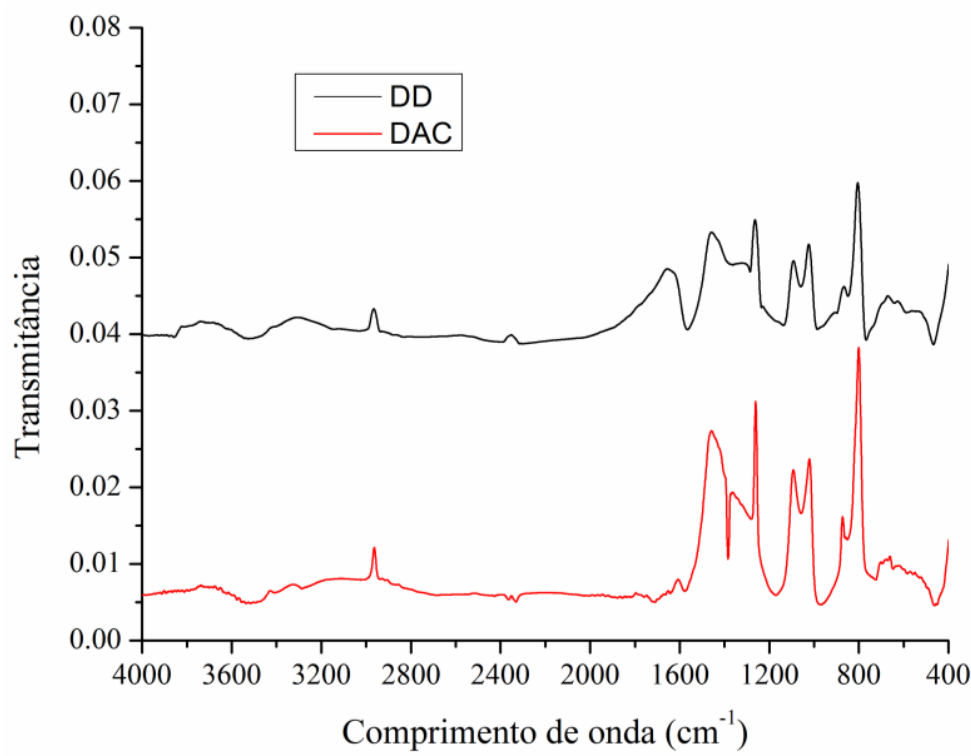

Figura 2: Espectroscopia no infravermelho dos carvões ativados de dendê in natura (DD) e funcionalizado DAC).

Foram observadas bandas nos espectros no infravermelho dos carvões na região de $3650-3200 \mathrm{~cm}^{-1}$, indicando presença de $-\mathrm{OH}$, normalmente associada à ligação hidrogênio, como destacado por Silverstein [22]. Esta absorção foi atribuída à presença de $(-\mathrm{OH})$ de grupos carboxílicos dos carvões ativados, caracterizados por forte absorção nesta mesma faixa [23]. Tais grupos podem estar relacionados aos ácidos carboxílicos detectados pelo método de Boehm, uma vez que não foram encontradas bandas referentes aos grupos carboxílicos $(\mathrm{C}=\mathrm{O})$, relativos à banda próxima a $1.700 \mathrm{~cm}^{-1}$. 
A banda a $1580 \mathrm{~cm}^{-1}$ (faixa entre $1700-1500 \mathrm{~cm}^{-1}$ ), visualizada nas amostras DD e DAC, pode estar associada ao estiramento do anel aromático acoplado ao grupo carbonila e ao aumento dos estiramentos das ligações $\mathrm{C}=\mathrm{O}$. A funcionalização com ácido nítrico promoveu aparecimento de banda a $1385 \mathrm{~cm}^{-1}$, correspondente às vibrações das bandas de $-\mathrm{NO}_{2}\left(1385-1325 \mathrm{~cm}^{-1}\right)$. De acordo com Gómez-Serrano [18], as alterações produzidas na química da superfície do carbono por meio de tratamento com $\mathrm{HNO}_{3}$ são devido à formação de vários grupos de superfície do oxigênio e das estruturas que contêm ligações N-O (-nitro grupos e complexos de nitrato).

$\mathrm{Na}$ faixa de 1200-1000 $\mathrm{cm}^{-1}$ as bandas indicam presença de grupos C-O que são características de grupos éteres, fenóis e hidroxilas. A banda próxima a $780 \mathrm{~cm}^{-1}$ foi atribuída a vibrações de éster e anéis aromáticos monossubstituídos pela presença de lignina na matéria-prima [24].

\subsubsection{Ensaios Cinéticos}

Os dados experimentais relativos à cinética e os ajustes dos modelos são apresentados na Figura 3.
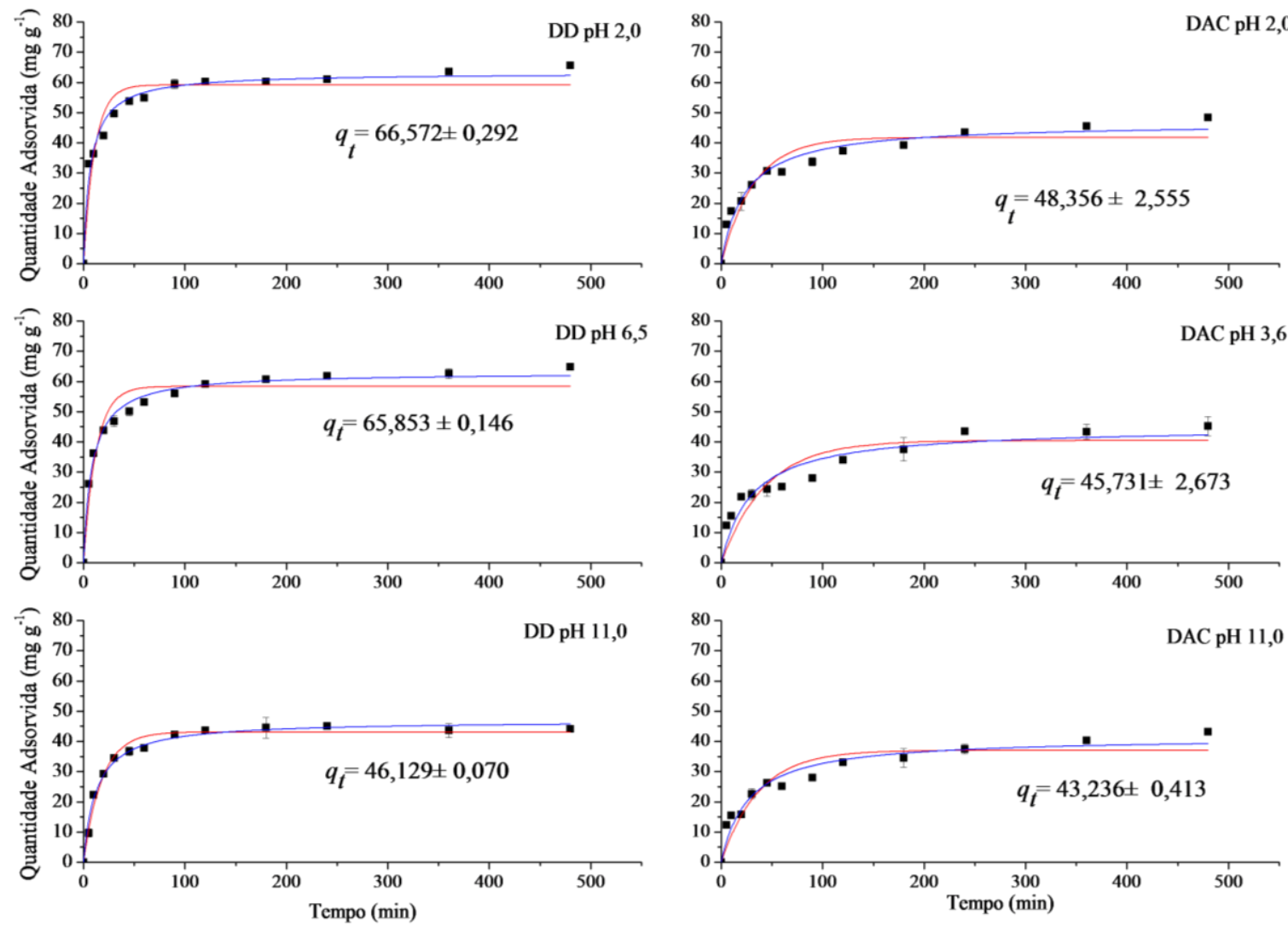

Figura 3: Cinética de adsorção do paracetamol em carvão DD e DAC em diferentes valores de pH ( - pseudo-primeira ordem, - pseudo-segunda ordem, dados experimentais).

Observa-se a partir dos dados cinéticos que a taxa de adsorção é mais rápida nos primeiros 60 minutos e que após este tempo a quantidade adsorvida aumenta lentamente até o tempo de equilíbrio em 120 minutos para DD e de 200 minutos para DAC. Como verificado na Tabela 1, as áreas superficiais diminuíram devido ao bloqueio dos poros pela funcionalização em meio ácido, o que deve ter causado uma barreira na adsorção do paracetamol aumentando o tempo para alcance do equilíbrio comparado ao carvão ativado in natura.

A funcionalização com $\mathrm{HNO}_{3}$ promoveu diminuição da eficiência de remoção do paracetamol, com valores de $66,62 \%, 60,07 \%, 53,44 \%, 40,38 \%, 36,95 \%$ e $11,65 \%$ para $\mathrm{pH} 2,0, \mathrm{pH}_{\mathrm{PCZ}}$ e 11,0 para as amostras DD e DAC, respectivamente.

Nota-se que em pH 11,0 a quantidade adsorvida foi menor para ambos os carvões, o que pode ser explicado pela forma molecular do paracetamol. A molécula neutra ocorre na faixa de $\mathrm{pH}$ de 2,0 a 10,0. A partir daí, a molécula assume caráter aniônico devido à presença de carga negativa nos grupos fenólicos. Por outro lado, a superfície dos adsorventes em $\mathrm{pH}<\mathrm{pH}_{\mathrm{PCZ}}$ possui carga superficial positiva e em $\mathrm{pH}>\mathrm{pH} \mathrm{PCZ}_{\mathrm{O}}$ 
adsorventes apresentaram carga superficial negativa, ocorrendo, portanto, repulsão eletrostática em $\mathrm{pH}$ 11,0 [7].

Na Tabela 3 são apresentados os parâmetros cinéticos para DD e DAC, valores de $\mathrm{R}^{2}$ e de desvio padrão.

Tabela 3: Parâmetros cinéticos de pseudo-primeira ordem e pseudo-segunda ordem para adsorção do paracetamol em carvão ativado DD e DAC em $\mathrm{pH} 2,0, \mathrm{pH}_{\mathrm{PCZ}}$ e 11,0 .

\begin{tabular}{c|c|ccc}
\hline \multirow{3}{*}{ Amostra } & $\mathbf{p H}$ & \multicolumn{3}{c}{ Pseudo-primeira ordem } \\
& & $q_{\text {eq }}$ & $k_{l}\left(\mathrm{~h}^{-1}\right)$ & $R^{2}$ \\
\hline \multirow{4}{*}{ DD } & $\mathbf{2 , 0}$ & $59,113 \pm 1,892$ & $0,092 \pm 0,016$ & 0,912 \\
\cline { 2 - 5 } & $\mathbf{6 , 5}$ & $58,316 \pm 1,756$ & $0,081 \pm 0,013$ & 0,929 \\
\cline { 2 - 5 } & $\mathbf{1 1 , 0}$ & $43,077 \pm 0,797$ & $0,056 \pm 0,005$ & 0,980 \\
\hline \multirow{4}{*}{ DAC } & $\mathbf{2 , 0}$ & $41,748 \pm 2,008$ & $0,031 \pm 0,006$ & 0,896 \\
\cline { 2 - 5 } & $\mathbf{3 , 6}$ & $41,234 \pm 2,304$ & $0,031 \pm 0,006$ & 0,855 \\
\cline { 2 - 5 } & $\mathbf{1 1 , 0}$ & $37,002 \pm 2,080$ & $0,027 \pm 0,006$ & 0,868 \\
\hline \multirow{5}{*}{ DD } & & & Pseudo-segunda ordem & $R^{2}$ \\
\cline { 2 - 5 } & $\mathbf{2 , 0}$ & $63,111 \pm 1,244$ & $k_{2}\left(\mathrm{~g} \mathrm{mg}^{-1} \mathrm{~h}^{-1}\right)$ & 0,976 \\
\cline { 2 - 5 } & $\mathbf{6 , 5}$ & $62,915 \pm 0,929$ & $0,002 \pm 3,31 \times 10^{-4}$ & 0,988 \\
\hline \multirow{4}{*}{ DAC } & $\mathbf{1 1 , 0}$ & $46,81 \pm 0,804$ & $0,002 \pm 1,792 \times 10^{-4}$ & 0,988 \\
\cline { 2 - 5 } & $\mathbf{2 , 0}$ & $48,580 \pm 1,710$ & $6,164 \times 10^{-4} \pm 1,715 \times 10^{-4}$ & 0,961 \\
\cline { 2 - 5 } & $\mathbf{3 , 6}$ & $45,173 \pm 2,105$ & $0,001 \pm 2,527 \times 10^{-4}$ & 0,943 \\
\cline { 2 - 5 } & $\mathbf{1 1 , 0}$ & $44,385 \pm 2,015$ & $4,038 \times 10^{-4} \pm 2,169 \times 10^{-4}$ & 0,948 \\
\hline
\end{tabular}

Pela Figura 3 e pela Tabela 3 observa-se que o modelo de pseudo-segunda ordem se ajusta melhor aos dados experimentais, ou seja, a quimissorção possui contribuição significativa neste processo. A quantidade adsorvida experimental (Figura 3) foi similar ao valor obtido pelo modelo de pseudo-segunda ordem (Tabela 3). Verifica-se que o valor de $\mathrm{k}_{2}$ é menor para o DAC comparado ao valor para DD, provavelmente pela interferência da repulsão eletrostática, uma vez que o DAC possui maior quantidade de grupos ácidos (Tabela 2). De acordo com [10], a molécula do paracetamol, sendo fracamente ácida $(\mathrm{pKa}=9,9)$, interage principalmente com grupos básicos da superfície do carvão.

\subsection{Isotermas}

Os gráficos da isoterma de adsorção do paracetamol em carvões DD e DAC e as curvas obtidas pelo ajuste dos modelos de Langmuir, Freundlich e Sips são apresentados na Figura 4. 

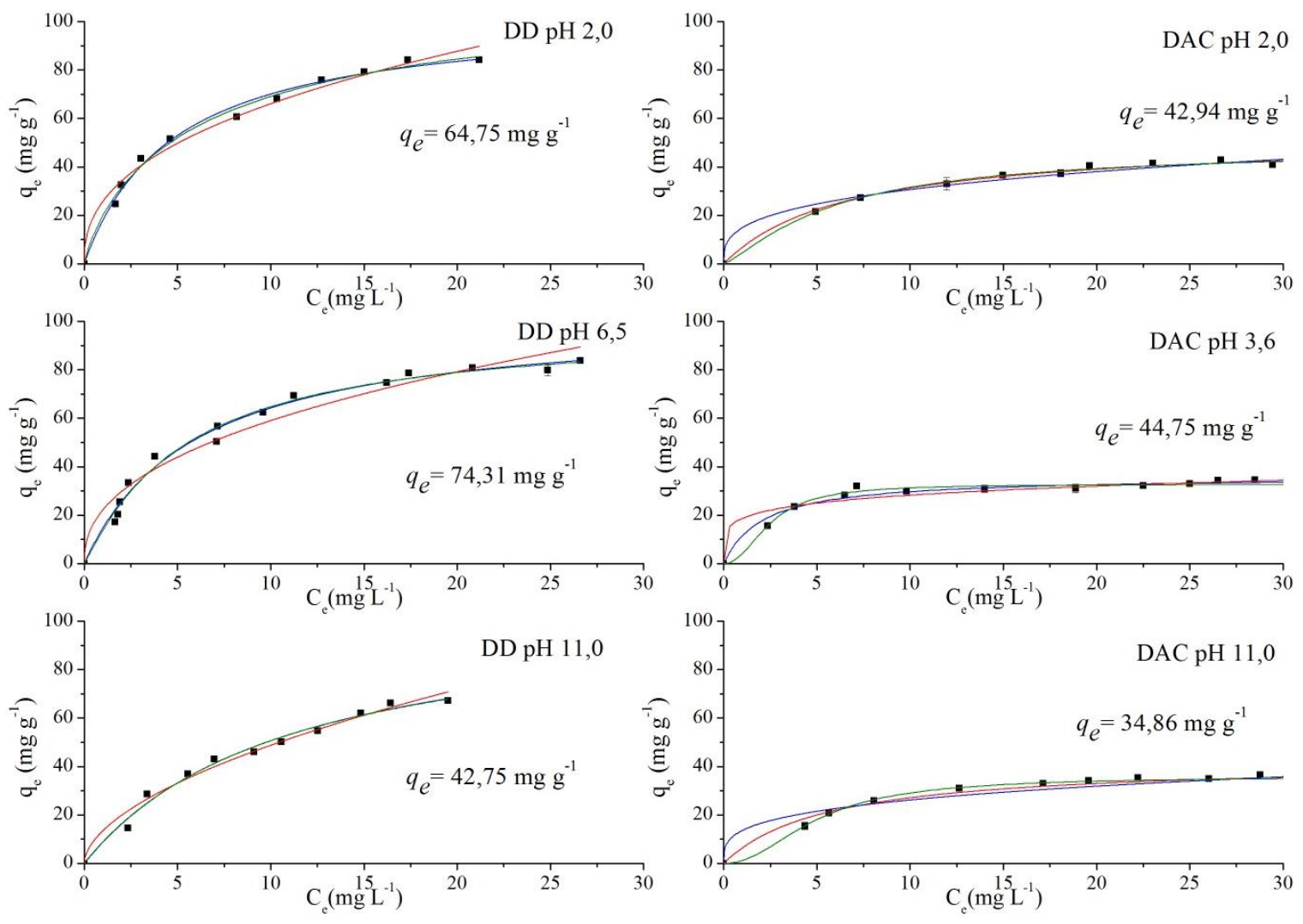

Figura 4: Isotermas de adsorção do paracetamol em carvões ativados DD e DAC em diferentes valores de $\mathrm{pH}$ (Langmuir, - Frendlich, - Sips, $\square$ dados experimentais).

Os carvões ativados DD e DAC apresentaram valores de capacidade de adsorção do paracetamol próximos aos valores de $q_{\text {máx }}$ de Langmuir para o DD e de Sips para DAC, e com maiores quantidades adsorvidas no $\mathrm{pH}_{\mathrm{PCZ}}$. A capacidade máxima do $\mathrm{DAC}$ foi menor do que a do $\mathrm{DD}$ para os valores de $\mathrm{pH}$ devido à presença de maiores quantidades de grupos ácidos nas superfícies dos carvões, como discutido nas cinéticas de adsorção (Tabela 3). 
Tabela 4: Parâmetros das isotermas de Langmuir, Freundlich e Sips em carvões ativados in natura e funcionalizado para $\mathrm{pH} 2,0, \mathrm{pH}_{\mathrm{PCZ}}$ e 11,0 .

\begin{tabular}{|c|c|c|c|c|c|c|}
\hline \multirow{2}{*}{ Amostra } & \multirow{2}{*}{$\mathbf{p H}$} & \multicolumn{5}{|c|}{ Langmuir } \\
\hline & & $q_{\max }$ & $k_{L}$ & $R^{2}$ & $\chi^{2}$ & \\
\hline \multirow{3}{*}{ DD } & 2,0 & $70,620 \pm 1,599$ & $0,280 \pm 0,026$ & 0,980 & 4,317 & \\
\hline & 6,5 & $90,813 \pm 3,073$ & $0,187 \pm 0,019$ & 0,984 & 5,735 & \\
\hline & 11,0 & $62,911 \pm 1,875$ & $0,117 \pm 0,029$ & 0,925 & 1,831 & \\
\hline \multirow{5}{*}{ DAC } & 2,0 & $51,829 \pm 1,231$ & $0,152 \pm 0,014$ & 0,993 & 13,77 & \\
\hline & 3,6 & $53,889 \pm 0,512$ & $0,149 \pm 0,005$ & 0,999 & 7,683 & \\
\hline & 11,0 & $42,069 \pm 1,601$ & $0,130 \pm 0,028$ & 0,997 & 5,934 & \\
\hline & & \multicolumn{5}{|c|}{ Freundlich } \\
\hline & & $k_{F}$ & $1 / n$ & $R^{2}$ & $\chi^{2}$ & \\
\hline \multirow{3}{*}{ DD } & 2,0 & $25,843 \pm 1,937$ & $0,281 \pm 0,349$ & 0,948 & 1,128 & \\
\hline & 6,5 & $21,389 \pm 2,209$ & $0,404 \pm 0,235$ & 0,944 & 0,206 & \\
\hline & 11,0 & $12,212 \pm 2,388$ & $0,422 \pm 0393$ & 0,879 & 3,214 & \\
\hline \multirow{5}{*}{ DAC } & 2,0 & $14,914 \pm 1,473$ & $0,311 \pm 0,333$ & 0,976 & 3,757 & \\
\hline & 3,6 & $15,294 \pm 1,068$ & $0,314 \pm 0,229$ & 0,988 & 2,038 & \\
\hline & 11,0 & $13,562 \pm 1,928$ & $0,284 \pm 0,571$ & 0,930 & 7,873 & \\
\hline & & \multicolumn{5}{|c|}{ Sips } \\
\hline & & $q_{\max }$ & $k_{L}$ & $n$ & $R^{2}$ & $\chi^{2}$ \\
\hline \multirow{3}{*}{ DD } & 2,0 & $117,536 \pm 18,170$ & $0,152 \pm 0,062$ & $0,844 \pm 0,143$ & 0,991 & 6,431 \\
\hline & 6,5 & $118,158 \pm 7,576$ & $0,185 \pm 0,034$ & $1,073 \pm 0,131$ & 0,985 & 10,418 \\
\hline & 11,0 & $104,557 \pm 26,528$ & $0,094 \pm 0,049$ & $1,015 \pm 0,217$ & 0,985 & 6,792 \\
\hline \multirow{3}{*}{ DAC } & 2,0 & $47,474 \pm 2,391$ & $0,172 \pm 0,014$ & $1,269 \pm 0,194$ & 0,993 & 1,010 \\
\hline & 3,6 & $52,726 \pm 0,661$ & $0,406 \pm 0,029$ & $2,160 \pm 0,393$ & 0,978 & 2,054 \\
\hline & 11,0 & $35,697 \pm 0,673$ & $0,202 \pm 0,007$ & $2,096 \pm 0,265$ & 0,991 & 1,000 \\
\hline
\end{tabular}

O modelo de Langmuir obteve melhores ajustes dos dados experimentais, como pode ser verificado pelos valores dos coeficientes de determinação $\left(\mathrm{R}^{2}\right)$ e com menores valores da análise não linear pelo teste do qui-quadrado $(\chi 2)$ para o carvão DD. Para o carvão DAC o modelo de Sips obteve melhores ajustes dos dados experimentais, com valores de $\mathrm{R}^{2}$ próximos ao modelo de Langmuir, no entanto, com menores valores de $\chi 2$. No modelo de Sips o coeficiente $n$ é um indicativo da heterogeneidade da superfície, sendo mais heterogênea quanto mais próximo de zero for o valor deste parâmetro. Os valores obtidos $(>0,844)$ indicam maior homogeneidade da superfície. O resultado do modelo de Sips para DAC indica que em baixas concentrações de paracetamol, os dados experimentais seguem o modelo de Freundlich, enquanto que, para concentrações elevadas de paracetamol, a capacidade de adsorção se caracteriza com o modelo de Langmuir.

$\mathrm{O}$ modelo de Langmuir está associado à adsorção homogênea e em monocamada, característica de processos químicos que denotam maior seletividade na adsorção. Em ensaios de dessorção do paracetamol em carvões ativados, Couto Jr. [7] observou que os agentes regenerantes (água deionizada, $\mathrm{NaOH} \mathrm{2,5} \mathrm{mol} \mathrm{L}{ }^{-1}$, metanol $50 \% \mathrm{v} / \mathrm{v}$, etanol $50 \%$ v/v e etanol $94 \%$ v/v) não promoveram remoção completa do fármaco adsorvido, indicando que a dessorção está associada à presença de fortes interações, características da quimissorção do paracetamol em sítios superficiais do carvão ativado. Estes agentes regenerantes não foram capazes de romper estas fortes interações, demonstrando menor capacidade de dessorção.

Para Guilarduci [23] a funcionalização do carvão ativado com ácido sulfúrico (1 mol L $\left.\mathrm{L}^{-1}\right)$ aumenta o índice de heterogeneidade, indica menores tamanhos de poros e dificulta a entrada de fenol nos microporos; e para Ania [24] a presença de grupos funcionais ácidos na superfície impede a adsorção de compostos fenólicos nos carvões ativados. A retenção de alguns compostos orgânicos é menor em carvões ácidos do que em carvões de natureza básica, até mesmo quando sua área específica é maior [24].

\section{CONCLUSÕES}

$\mathrm{O}$ carvão ativado estudado apresenta química superficial neutra. A funcionalização com $\mathrm{HNO}_{3}$ diminuiu a área superficial e aumentou a quantidade de grupos ácidos, ocasionando diminuição no ponto de carga zero. 
O modelo cinético de pseudo-segunda ordem para ambos os carvões, o modelo de equilíbrio de Langmuir para DD e o modelo de equilíbrio de Sips para DAC foram os que melhor ajustaram os dados experimentais em todas as amostras.

A maior quantidade adsorvida do paracetamol nas amostras foi verificada em $\mathrm{pH} 2,0$ e $\mathrm{pH}_{\mathrm{PCZ}}$. Em pH 11,0 as amostras apresentaram carga superficial negativa $\left(\mathrm{pH}>\mathrm{pH}_{\mathrm{PCZ}}\right)$, ocorrendo repulsão eletrostática entre o paracetamol (forma aniônica) e os adsorventes, o que justifica os piores rendimentos. $\mathrm{Em} \mathrm{pH}<\mathrm{pH} \mathrm{PCZ}_{\mathrm{a}}$ carga é positiva no adsorvente e neutra para o paracetamol, podendo ocorrer atração entre os grupos básicos e a carbonila do paracetamol, indicando que a quimissorção pode estar envolvida no processo.

$\mathrm{O}$ carvão funcionalizado com $\mathrm{HNO}_{3}$ diminuiu a quantidade adsorvida do paracetamol, demonstrando a interferência dos grupos ácidos na eficiência de remoção deste fármaco.

\section{AGRADECIMENTOS}

Os autores agradecem a Capes e ao CNPq pela concessão da bolsa de mestrado e de doutorado para a Eng. Regiane Cristina Ferreira.

\section{BIBLIOGRAFIA}

[1] TRAN, N.H., LI, J., HU, J., ONG, S.L. "Occurrence and suitability of pharmaceuticals and personal care products as molecular markers for raw wastewater contamination in surface water and groundwater", Environmental Science and Pollution Research, v.21, n.6, pp.4727-4740, 2014.

[2] SHANMUGAM, G., SAMPATH, S., SELVARAJ, K. K., et al., "Non-steroidal anti-inflammatory drugs in Indian rivers", Environmental Science and Pollution Research, v. 21, pp. 921-31, 2014.

[3] ASHFAQ, M., NAWAZ KHAN, K., SAIF UR REHMAN, M., et al., "Ecological risk assessment of pharmaceuticals in the receiving environment of pharmaceutical wastewater in Pakistan", Ecotoxicology and Environmental Safety, v. 136, pp. 31-9, 2017.

[4] MELO, S. A. S., TROVÓ, A. G., BAUTITZ, I. R., et al., "Degradação de fármacos residuais por processos oxidativos avançados," Química Nova, v. 32, pp. 188-197, 2009.

[5] GALHETAS, M., ANDRADE, M.A., MESTRE, A.S., et al., "The influence of the textural properties of activated carbons on acetaminophen adsorption at different temperatures", Physical Chemistry Chemical Physics (PCCP), v.17, n.18, pp.12340-12349, 2015.

[6] COUTO JUNIOR, O. M., MATOS, I., CARVALHO, D. S., et al., "Effects of pH and activated carbon surface chemistry on the adsorption of caffeine from aqueous solution", In: X Encontro Brasileiro sobre Adsorção, 1-8, Guarujá, São Paulo, Brasil, 27-30 Abril 2014.

[7] COUTO JUNIOR, O. M. Adsorção de ibuprofeno e paracetamol em carvão ativado funcionalizado, Tese de D.Sc.,DEQ/UEM, Maringá, PR, Brasil, 2014.

[8] PARK, J., REGALBUTO, J. R. "A simple, accurate determination of oxide PZC and the strong buff-ering effect of oxide surfaces at incipient wetness", Journal of Colloid and Interface Science, v. 175, n.1, pp. 239252,1995

[9] BOEHM, H., "Surface oxides on carbon and their analysis: a critical assessment", Carbon, v. 40, n.2, pp. 145-149, 2002.

[10] FERREIRA, R. C., COUTO JUNIOR, O. M., CARVALHO, K. Q., et al., "Effect of Solution pH on the Removal of Paracetamol by Activated Carbon of Dende Coconut Mesocarp", Chemical and Biochemical Engineering Quarterly Journal, v. 29, n.1, pp. 47-53, 2015.

[11] LAGERGREN S., "Zur Theorie der sogenannten Adsorption gelöster Stoffe", K. Sven. vetenskapsakademiens, Handl., v. 24, pp. 1-39, 1898.

[12] HO Y., MCKAY, G., "Pseudo-second order model for sorption processes", Process Biochemistry, v. 34, n.5, pp. 451-465, 1999.

[13] LANGMUIR, I., "The constitution and fundamental properties of solids and liquids", Journal of the American Chemical Society, v. 38, n.11, pp. 2221-95, 1916.

[14] RUTHVEN, D. M., Principles of Adsorption and Adsorption Processes, John Wiley. 1984.

[15] SIPS, R. "On the structure of a catalyst surface", The Journal of Chemical Physics. v. 16, pp. 490-495, 1948.

[16] THOMMES, M., KANEKO, K., NEIMARK, A. V., et al., "Physisorption of gases, with special reference to the evaluation of surface area and pore size distribution (IUPAC Technical Report)", Pure and 
Applied Chemistry. v. 87, pp. 1051-1069, 2015.

[17] LOPES A. S. D. C., Modificação da superfície de carvão ativado comercial (CAG) para a aplicação na adsorção de benzeno e tolueno, Dissertação de Ms. PPGEQ / UFPA, Belém, Pará, Brasil, 2012.

[18] GÓMEZ-SERRANO, V., ACEDO-RAMOS, M., LÓPEZ-PEINADO, A. J., et al., "Mass and surface changes of activated carbon treated with nitric acid. Thermal behavior of the samples", Thermochimica Acta., v. 291, n.1-2, pp. 109-115, 1997.

[19] SHIM, J. W., PARK, S. J., RYU, S. K., "Effect of modification with HNO3 and NaOH on metal adsorption by pitch-based activated carbon fibers", Carbon, v. 39, pp. 1635-1642, 2001.

[20] FERNANDES, F. L., Carvão de endocarpo de coco da baía ativado quimicamente com ZnCl2 e fisicamente com vapor d'água: produção, caracterização, modificações químicas e aplicação na adsorção de ín cloreto, Tese de D.Sc., CCEN/UFPB, João Pessoa, Paraíba, Brasil, 2008.

[21] JULIEN, F., BAUDU, M., MAZET, M. "Relationship Between Chemical and Physical Surface Properties of Activated Carbon", Water Research, v. 32, pp. 3414-3424, 1998.

[22] SILVERSTEIN, R. M., WEBSTER, F. X., KIEMLE, D. J. Spectrometric Identification of Organic Compounds, 7th ed. John Wiley \& Sons, 2005.

[23] GUILARDUCI, V. V. D. S., MESQUITA, J. P., MARTELLI, P. B., et al., "Adsorção de fenol sobre carvão ativado em meio alcalino", Química Nova, v. 29, pp. 1226-1232, 2006.

[24] ANIA, C. O., PARRA, J. B., PIS, J. J. "Effect of texture and surface chemistry on adsorptive capacities of activated carbons for phenolic compounds removal", Fuel Processing Technology, v. 77-78, pp. 337-343, 2002. 